\title{
FINDING AGES FOR OLD STELLAR POPULATIONS
}

\author{
GUY WORTHEY \\ Department of Astronomy \\ University of Michigan \\ Ann Arbor, MI 48109-1090
}

We are far from being able to populate a histogram of star formation versus time for an elliptical galaxy based solely on observations of its spectrum, but the path toward such a dream is becoming more clear. Still, we are denied the easiest paths, and most of what I have been thinking about in the last six months are the obstacles rather than the opportunities in age determinations for old stellar populations, and Es in particular. The following list should illustrate what I mean.

\section{3/2 degeneracy: we need abundance to get age}

Age effects and metallicity effects have similar effects on broad-band colors and spectral features. Aaronson et al. (1978) found that this degeneracy followed the (approximate) rule $\delta \log$ Age $/ \delta \log Z \approx 3 / 2$ and this has been confirmed by subsequent studies. Thus a population 15 Gyr old but a little less than solar abundance mimics almost perfectly the colors and line strengths of a 5 Gyr population that is twice as metal-rich. The immediate implication is that one needs to know the abundance to $7 \%$ in order to get a $10 \%$-accuracy age estimate.

\section{3/2 degeneracy: colors alone are not adequate}

Since the usual case is that one knows neither the age nor the abundance at first, it is needful to find spectral indices that are dependent on only one parameter. Broad-band colors simply do not fit this description, and neither do most prominent absorption features. Worthey (1994) points out some indices which are relatively more age-sensitive than the average (Balmer lines) and some indices which are relatively more $Z$-sensitive. Taken in maximally contrasting pairs, the indices can be used to find a simultaneous "mean age" and "mean $Z$," both of which must be regarded as heuristic at 
this point in time. Work on finding more and better age- and $Z$-sensitive indices should command a high priority. To date, we have not explored much of what the spectrum can offer us.

\section{3. model age uncertainties are $\pm 35 \%$}

Charlot, Worthey, \& Bressan (1996) have recently compared different synthesis models to find that the author-to-author spread in predictions correspond to an age error of $\pm 35 \%$. This spread comes from models with the same input metallicity, age, and IMF, and so is independent of any astrophysical uncertainties. Uncertainties in both the input stellar evolution and the assumed stellar fluxes contribute to this error. The $35 \%$ uncertainty extends also to Balmer-metal diagrams. The error can be reduced over time, but the process is not straightforward due to its complexity.

\section{4. abundance ratios in real galaxies are not solar}

O'Connell (1976) noticed that solar-neighborhood stars could not, in any combination, match the absorption features of giant ellipticals. Features due to $\mathrm{Mg}, \mathrm{Na}$, and $\mathrm{CN}$ are too strong compared to Fe blends (Worthey 1994). Since the deviations do not mimic age- $Z$ degeneracy, variations in element-to-element abundance ratios are unambiguously implicated; giant Es have an abundance pattern more suggestive of halo stars than of stars in the solar neighborhood.

Why is this pertinent? Abundance ratio changes add a new level of complexity to age determinations because elemental mixture determines isochrone shape and stellar lifetimes, which directly affect age estimates. Furthermore, the two most important elements, Oxygen and Helium, don't have observable absorption features in the spectra of old populations, and so promise to be extremely difficult to calibrate. We already know that we cannot simply extrapolate the halo mix to higher metallicities because $\mathrm{Na}$ is enhanced in Es, but not in the halo. Furthermore, our Galactic bulge appears to have $[\mathrm{O} / \mathrm{Fe}]$ nearly solar, again bucking the halo trend (Rich, this volume).

Even more pertinent, abundance ratio changes mean that a derived "mean age" will be a function of the elemental species used as the metal indicator. For example, using Lick/IDS data and Worthey models in Balmermetal diagrams, ellipticals plotted versus Fe features look old and of nearly solar composition. However, plotted versus $\mathrm{Mg}_{2}$, they look extremely metalrich (maybe +0.75 to +1.0 ) and only $1-2$ Gyr old. I am eager to see how the challenge of the abundance ratio dilemma will be met in years to come. 


\section{Balmer absorption is often filled by nebular emission}

An observational difficulty with the current-best age indicators is that the stellar Balmer absorption can be partially or completely filled in by nebular emission.

\section{6. populations that are composite in age and metallicity}

Although it is now possible to invert a Balmer-metal diagram via models to obtain a "mean age" that doesn't mean it is a particularly good idea, since the "mean age" thus obtained is very strongly weighted toward any young populations that might be present. A 1-Gyr population has Balmer lines a factor of 5 stronger and a luminosity more than 6 times greater than a $15 \mathrm{Gyr}$ population, so only a few percent by mass of a young population will dominate the age estimate.

Another aspect of composite populations that will cause trouble in the future is that the more sensitive metal lines probe the more metal-rich populations (since the lines are stronger for those populations) but Balmer lines and other age indicators sensitive to turnoff color will be more sensitive to the metal-poor subpopulations because they contain more warm stars. This is calibratable if one assumes a chemical enrichment history, but it looks like a difficult chore to derive it from the galaxy spectrum itself.

In summary, we find ourselves at the "mean age" stage, but beset by fairly serious problems, the most serious of which are instrinsic model unreliability and the whole new world of altered abundance ratios in Es. The next steps are (1) to explore age sensitivity in the spectrum to a greater extent, (2) to start a program of evolutionary models which take into account many different abundance mixes, (3) to begin to explore compositness, and (4) to utilize empirical feedback to improve overall model reliability.

\section{References}

Aaronson, M., Cohen, J. G., Mould, J. R., \& Malkan, M. 1978, ApJ, 223, 824

Charlot, S., Worthey, G., \& Bressan, A. 1996, ApJ, in press, Feb. 1

González, J. J. 1993, Ph.D. Thesis, Univ. of California, Santa Cruz

O'Connell, R. W. 1976, ApJ, 206, 370

Worthey, G. 1994, ApJS, 95, 107

\section{DISCUSSION}

O. Gerhard: Could you give me an indication of the spread of main sequence turnoff ages for $1 M_{\odot}$ stars as a function of metallicity and elemental ratios?

G. Worthey: A $1 M_{\odot}$ turnoff at $[\mathrm{Fe} / \mathrm{H}] \approx-1.0$ is about $8 \mathrm{Gyr}$, at $[\mathrm{Fe} / \mathrm{H}]$ $\approx 0$ is about $12 \mathrm{Gyr}$. To a first approximation, if you change the elemental 
ratios the stellar lifetimes are approximately what you would get if you added all the metals up and forced a solar mix to equal that total. The details here are crying out for some good modeling. Above solar metallicity, helium abundance has a large impact on lifetime.

O. Gerhard: In some ellipticals, two mean metallicities have been assigned to their globular clusters, suggesting a merger history. If you took two stellar populations with these metallicities and related SF peaks what mean metallicity and age would you find?

G. Worthey: As a first cut, weight by $V$-band flux.

B. Dorman: Your plot [mean age versus mean abundance with galaxy index data from González 1993 inverted via Worthey models] puts M31 in a very strange location, with a similar age for M32, but much more metalrich. My poster has a fit to M31 with age $10 \mathrm{Gyr}$ and solar metallicity. The mid-UV flux strength (not dependent on any assumptions about the origin of the UV upturn) excludes a high metallicity except for very young ages. G. Worthey: First, the age and metallicity zeropoints from the Balmermetal diagrams are unknown, so from that perspective our conclusions about M31 are in accord. For large Es, about solar metallicity is what one derives from broad band colors assuming old ages. Second, the IUE data you use has a $10 \times 20$ " aperture compared to ground based spectrographs which have something like a $1-2$ " aperture. The González aperture is just large enough to get both the dynamical nucleus and the higher surface brightness interloper nucleus, so there is probably a bigger problem than just an aperture effect to remove before we are talking about the same target population. Third, your age-metallicity pair is along the $3 / 2$ degeneracy slope from M31 in my figure. Fourth, I do not think that we can yet discount a youthful component or an underlying ordinary metal-poor-withhot-horizontal branch component in (the spiral galaxy) M31 as significant contributors to the 1500-2500 $\AA$ flux. Fifth, based on abundances in our own Galactic bulge, one would naively expect to find nuclear populations in M31 which are greater than solar $Z$, if not $[\mathrm{Fe} / \mathrm{H}]$.

B. Dorman: The galaxies with the strongest UVX appear to have a large age spread.

B. M. Poggianti: Studying $\mathrm{H} \delta$ strength in single stars and simple stellar populations using Kurucz's models we found that only warm stars have strong Balmer lines. For solar metallicities, only main sequence stars are placed in this "high Balmer region," but in metal poor populations, some horizontal branch stars populate that region. Balmer lines do not depend on metallicity for a single star, but in integrated spectra they do depend on it. Can you exclude that this effect affects your interpretation of the $\mathrm{H} \beta$ line for ages of galaxies and for radial gradients within galaxies?

G. Worthey: Yes, excluding bizarre composite population effects. 